\title{
MolGPT: Molecular Generation using a Transformer-Decoder Model
}

\author{
Viraj Bagal, ${ }^{\dagger, \ddagger}$ Rishal Aggarwal, ${ }^{\dagger}$ P. K. Vinod, ${ }^{\dagger}$ and U. Deva Priyakumar ${ }^{*}, \dagger$ \\ $\dagger$ International Institute of Information Technology, Hyderabad 500 032, India \\ $\ddagger$ Indian Institute of Science Education and Research, Pune 411 008, India \\ E-mail: deva@iiit.ac.in
}

\section{List of Tables}

S1 Scaffold + Single property (logP, TPSA) conditional training on MOSES dataset. Metric calculated only for molecules having tanimoto similarity of the scaffold of the generated molecule and the scaffold used for condition greater than 0.8. (a) $\mathrm{O}=\mathrm{C}(\mathrm{Cc} 1 \mathrm{ccccc} 1) \mathrm{NCc} 1 \mathrm{ccccc} 1$ (b) c1cnc2[nH] $\operatorname{ccc} 2 \mathrm{c} 1$ (c) $\mathrm{c} 1 \mathrm{ccc}(-$ c2ccnnc2)cc1 (d) c1ccc(-n2cnc3ccccs32)cc1 (e) $\mathrm{O}=\mathrm{C}(\mathrm{c} 1 \mathrm{cc}[\mathrm{nH}] \mathrm{c} 1) \mathrm{N} 1 \mathrm{CCN}(\mathrm{c} 2 \mathrm{ccccc} 2) \mathrm{CC} 1 \mathrm{~S} 3$

S2 Scaffold + Multi-property conditional training on MOSES dataset. Metric calculated only for molecules having tanimoto similarity of the scaffold of the generated molecule and the scaffold used for condition greater than 0.8. (a) $\mathrm{O}=\mathrm{C}(\mathrm{Cc} 1 \mathrm{ccccc} 1) \mathrm{NCc} 1 \mathrm{cccc} 1 \quad$ (b) $\mathrm{c} 1 \mathrm{cnc} 2[\mathrm{nH}] \operatorname{ccc} 2 \mathrm{c} 1 \quad$ (c) $\operatorname{c1ccc}(-\mathrm{c} 2 \mathrm{ccnn} \mathrm{c} 2) \mathrm{cc} 1$ (d) $\mathrm{c} 1 \mathrm{ccc}(-\mathrm{n} 2 \mathrm{cnc} 3 \operatorname{ccccc} 32) \mathrm{cc} 1(\mathrm{e}) \mathrm{O}=\mathrm{C}(\mathrm{c} 1 \mathrm{cc}[\mathrm{nH}] \mathrm{c} 1) \mathrm{N} 1 \mathrm{CCN}(\mathrm{c} 2 \mathrm{ccccc} 2) \mathrm{CC} 1$.

\section{List of Figures}

S1 Scaffolds from test set used for scaffold + property based conditioning results. S5 
S2 Scaffold 1: $\mathrm{O}=\mathrm{C}(\mathrm{Cc} 1 \mathrm{ccccc} 1) \mathrm{NCc} 1 \mathrm{ccccc} 1$. Scaffold 2: c1cnc2[nH] ccc2c1. In both the subfigures, the molecule in black box is the scaffold used for conditional generation. (a, b) 8 random generated molecules having the same scaffold as scaffold 1 and 2 respectively. . . . . . . . . . . . . .

S3 Scaffold 1: $\mathrm{O}=\mathrm{C}(\mathrm{Cc} 1 \mathrm{ccccc} 1) \mathrm{NCc1} 1 \mathrm{ccccc}$. Scaffold 2: c1cnc2[nH]ccc2c1. In all the subfigures, the molecule in black box is the scaffold used for conditional generation. (a, b) Conditioned on scaffold as well as $\log \mathrm{P}=2 . \quad(\mathbf{c}, \mathbf{d})$ Conditioned on scaffold as well as SAS $=2.75 \ldots \ldots \ldots \ldots \ldots$ 
Table S1: Scaffold + Single property (logP, TPSA) conditional training on MOSES dataset. Metric calculated only for molecules having tanimoto similarity of the scaffold of the generated molecule and the scaffold used for condition greater than 0.8. (a) $\mathrm{O}=\mathrm{C}(\mathrm{Cc} 1 \mathrm{ccccc} 1) \mathrm{NCc} 1 \mathrm{ccccc} 1$ (b) c1cnc2[nH] $\mathrm{ccc} 2 \mathrm{c} 1$ (c) c1ccc(-c2ccnnc2) cc1 (d) c1 1 ccc(n2cnc3ccccc32)cc1 (e) $\mathrm{O}=\mathrm{C}(\mathrm{c} 1 \mathrm{cc}[\mathrm{nH}] \mathrm{c} 1) \mathrm{N} 1 \mathrm{CCN}(\mathrm{c} 2 \mathrm{ccccc} 2) \mathrm{CC} 1$

\begin{tabular}{|c|c|c|c|c|c|c|}
\hline Cond & Validity & Unique & Novelty & MAD & SD & SSF \\
\hline (a) $+\log \mathrm{P}$ & 0.99 & 0.512 & 1.0 & 0.122 & 0.116 & 0.993 \\
\hline (b) $+\log \mathrm{P}$ & 0.901 & 0.836 & 1.0 & 0.127 & 0.098 & 0.923 \\
\hline (c) $+\log \mathrm{P}$ & 0.940 & 0.627 & 1.0 & 0.129 & 0.11 & 0.960 \\
\hline (d) $+\log \mathrm{P}$ & 0.987 & 0.590 & 1.0 & 0.136 & 0.136 & 0.995 \\
\hline$(\mathrm{e})+\log \mathrm{P}$ & 0.991 & 0.228 & 1.0 & 0.114 & 0.115 & 0.995 \\
\hline (a) + SAS & 0.979 & 0.778 & 1.0 & 0.125 & 0.140 & 0.984 \\
\hline (b) $+\mathrm{SAS}$ & 0.912 & 0.629 & 1.0 & 0.141 & 0.122 & 0.929 \\
\hline (c) + SAS & 0.851 & 0.695 & 1.0 & 0.143 & 0.129 & 0.855 \\
\hline (d) $+\mathrm{SAS}$ & 0.969 & 0.717 & 1.0 & 0.135 & 0.189 & 0.973 \\
\hline (e) $+\mathrm{SAS}$ & 0.851 & 0.342 & 1.0 & 0.104 & 0.129 & 0.973 \\
\hline (a)+TPSA & 0.998 & 0.609 & 1.0 & 1.899 & 2.015 & 0.998 \\
\hline (b) + TPSA & 0.877 & 0.815 & 1.0 & 2.881 & 2.128 & 0.926 \\
\hline (c)+TPSA & 0.986 & 0.594 & 1.0 & 1.972 & 2.082 & 0.989 \\
\hline (d)+TPSA & 0.995 & 0.649 & 1.0 & 2.696 & 2.402 & 0.998 \\
\hline (e) + TPSA & 0.994 & 0.192 & 1.0 & 3.914 & 3.922 & 0.998 \\
\hline (a) + QED & 0.987 & 0.762 & 1.0 & 0.040 & 0.050 & 0.992 \\
\hline (b) + QED & 0.844 & 0.859 & 1.0 & 0.040 & 0.049 & 0.924 \\
\hline (c) $+\mathrm{QED}$ & 0.961 & 0.722 & 1.0 & 0.034 & 0.043 & 0.969 \\
\hline$(\mathrm{d})+\mathrm{QED}$ & 0.993 & 0.750 & 1.0 & 0.065 & 0.053 & 0.998 \\
\hline$(\mathrm{e})+\mathrm{QED}$ & 0.893 & 0.275 & 1.0 & 0.073 & 0.079 & 0.996 \\
\hline
\end{tabular}


Table S2: Scaffold + Multi-property conditional training on MOSES dataset. Metric calculated only for molecules having tanimoto similarity of the scaffold of the generated molecule and the scaffold used for condition greater than 0.8. $\mathrm{O}=\mathrm{C}(\mathrm{Cc} 1 \mathrm{ccccc} 1) \mathrm{NCc} 1 \mathrm{ccccc} 1 \quad$ (b) $\mathrm{c} 1 \mathrm{cnc} 2[\mathrm{nH}] \mathrm{ccc} 2 \mathrm{c} 1 \quad$ (c) $\mathrm{c} 1 \mathrm{ccc}(-\mathrm{c} 2 \mathrm{ccnn} \mathrm{c} 2) \mathrm{cc} 1 \quad$ (d) $\mathrm{c} 1 \mathrm{ccc}(-$ n2cnc3eccce32) cc1 (e) $\mathrm{O}=\mathrm{C}(\mathrm{c} 1 \mathrm{cc}[\mathrm{nH}] \mathrm{c} 1) \mathrm{N} 1 \mathrm{CCN}(\mathrm{c} 2 \mathrm{ccccc} 2) \mathrm{CC} 1$

\begin{tabular}{|c|c|c|c|c|c|c|}
\hline Cond & Validity & Unique & Novelty & $\begin{array}{c}\mathrm{MAD} / \mathrm{SD} \\
\mathrm{TPSA}\end{array}$ & $\begin{array}{c}\mathrm{MAD} / \mathrm{SD} \\
\log \mathrm{P}\end{array}$ & $\overline{\mathrm{SSF}}$ \\
\hline (a) $+\mathrm{TPSA}+\log \mathrm{P}$ & 0.961 & 0.489 & 1.0 & $3.133 / 3.955$ & $0.238 / 0.209$ & 0.988 \\
\hline (b) $+\mathrm{TPSA}+\log \mathrm{P}$ & 0.826 & 0.750 & 1.0 & $3.877 / 2.917$ & $0.169 / 0.149$ & 0.903 \\
\hline (c) $+\mathrm{TPSA}+\log \mathrm{P}$ & 0.963 & 0.606 & 1.0 & $3.526 / 2.709$ & $0.125 / 0.119$ & 0.971 \\
\hline (d) $+\mathrm{TPSA}+\log \mathrm{P}$ & 0.770 & 0.576 & 1.0 & $4.795 / 4.066$ & $0.164 / 0.172$ & 0.959 \\
\hline (e) $+\mathrm{TPSA}+\log \mathrm{P}$ & 0.995 & 0.262 & 1.0 & $3.680 / 3.999$ & $0.227 / 0.273$ & 0.995 \\
\hline Cond & Validity & Unique & - & $\begin{array}{c}\mathrm{MAD} / \mathrm{SD} \\
\mathrm{SAS}\end{array}$ & $\begin{array}{c}\mathrm{MAD} / \mathrm{SD} \\
\log \mathrm{P}\end{array}$ & $\mathrm{SSF}$ \\
\hline (a) $+\mathrm{SAS}+\log \mathrm{P}$ & 0.935 & 0.603 & 1.0 & $0.152 / 0.165$ & $0.237 / 0.256$ & 0.946 \\
\hline (b) $+\mathrm{SAS}+\log \mathrm{P}$ & 0.726 & 0.502 & 1.0 & $0.159 / 0.099$ & $0.164 / 129$ & 0.853 \\
\hline$(\mathrm{c})+\mathrm{SAS}+\log \mathrm{P}$ & 0.917 & 0.528 & 1.0 & $0.152 / 0.106$ & $0.161 / 171$ & 0.959 \\
\hline (d) $+\mathrm{SAS}+\log \mathrm{P}$ & 0.965 & 0.536 & 1.0 & $0.136 / 0.126$ & $0.191 / 0.194$ & 0.971 \\
\hline (e) $+\mathrm{SAS}+\log \mathrm{P}$ & 0.986 & 0.253 & 1.0 & $0.124 / 0.118$ & $0.166 / 0.194$ & 0.986 \\
\hline Cond & Validity & Unique & Novelty & $\begin{array}{c}\text { MAD/SD } \\
\text { TPSA }\end{array}$ & $\begin{array}{c}\mathrm{MAD} / \mathrm{SD} \\
\mathrm{SAS}\end{array}$ & SSF \\
\hline (a)+TPSA $+\mathrm{SAS}$ & 965 & 0.708 & 1.0 & $3.439 / 3.255$ & $0.179 / 0.174$ & 0.971 \\
\hline (b) $+\mathrm{TPSA}+\mathrm{SAS}$ & 0.822 & 0.558 & 1.0 & $4.711 / 3.093$ & $0.192 / 0.122$ & 0.842 \\
\hline (c) $+\mathrm{TPSA}+\mathrm{SAS}$ & 0.829 & 0.654 & 1.0 & $3.988 / 3.351$ & $0.181 / 0.124$ & 0.836 \\
\hline (d) $+\mathrm{TPSA}+\mathrm{SAS}$ & 0.943 & 0.668 & 1.0 & $4.010 / 3.424$ & $0.164 / 179$ & 0.946 \\
\hline (e) $+\mathrm{TPSA}+\mathrm{SAS}$ & 0.992 & 0.300 & 1.0 & $3.364 / 3.704$ & $0.145 / 0.128$ & 0.991 \\
\hline
\end{tabular}

\begin{tabular}{cccccccc}
\hline Cond & Validity & Unique & Novelty & $\begin{array}{c}\text { MAD/SD } \\
\text { TPSA }\end{array}$ & $\begin{array}{c}\text { MAD/SD } \\
\operatorname{logP}\end{array}$ & $\begin{array}{c}\text { MAD/SD } \\
\text { SAS }\end{array}$ & SSF \\
\hline (a)+TPSA+ $\log P+$ SAS & 0.799 & 0.423 & 1.0 & $5.095 / 5.241$ & $0.548 / 0.544$ & $0.355 / 0.378$ & 0.907 \\
(b)+TPSA+ $\log +$ SAS & 0.827 & 0.439 & 1.0 & $5.149 / 4.100$ & $0.226 / 0.189$ & $0.229 / 0.139$ & 0.844 \\
(c)+TPSA+logP+SAS & 0.352 & 0.427 & 1.0 & $5.814 / 6.477$ & $0.251 / 0.241$ & $0.219 / 181$ & 0.877 \\
(d)+TPSA+ + ogP+SAS & 0.284 & 0.429 & 1.0 & $5.258 / 5.662$ & $0.336 / 0.452$ & $0.246 / 0.311$ & 0.777 \\
(e)+TPSA+logP+SAS & 0.960 & 0.0 .214 & 1.0 & $5.858 / 5.360$ & $0.385 / 0.326$ & $0.236 / 229$ & 0.958 \\
\hline
\end{tabular}



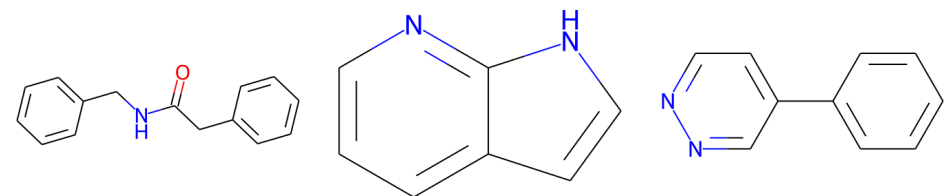

(a) Scaffold 1

(b) Scaffold 2

(c) Scaffold 3
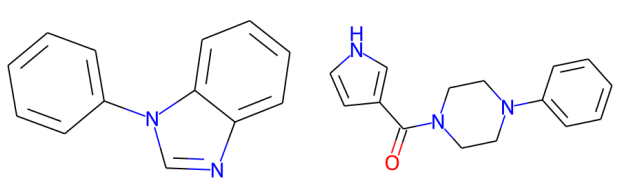

(d) Scaffold 4

(e) Scaffold 5

Figure S1: Scaffolds from test set used for scaffold + property based conditioning results.
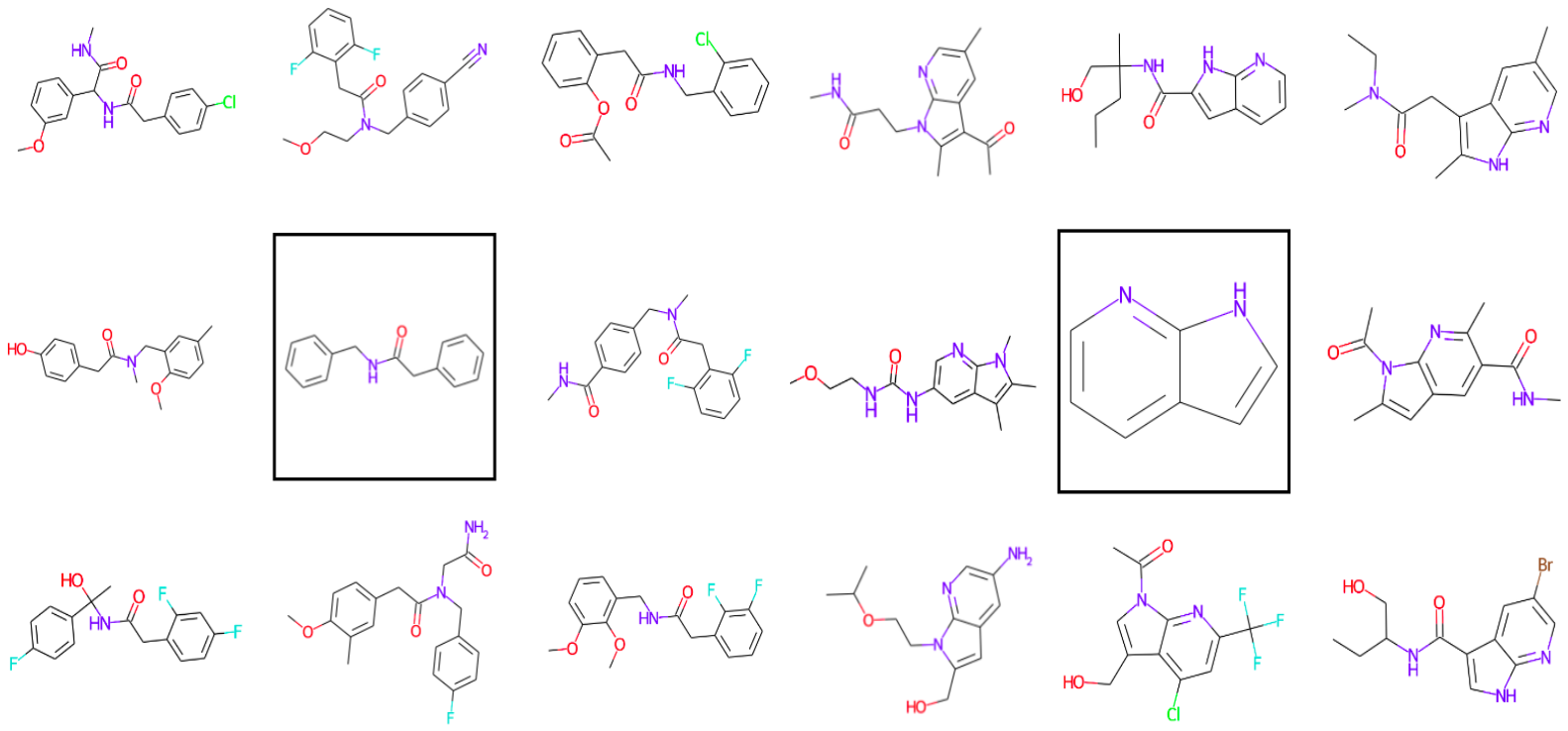

(a) Scaffold 1
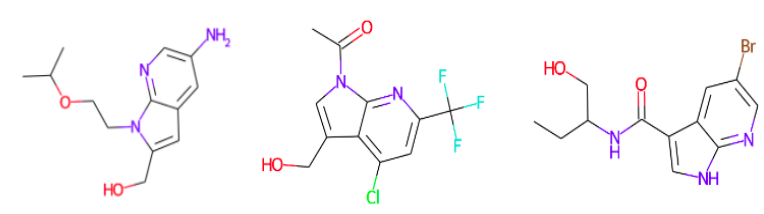

(b) Scaffold 2

Figure S2: Scaffold 1: $\mathrm{O}=\mathrm{C}(\mathrm{Cc} 1 \mathrm{ccccc} 1) \mathrm{NCc1}$ ccccc1. Scaffold 2: $\mathrm{c} 1 \mathrm{cnc} 2[\mathrm{nH}] \mathrm{ccc} 2 \mathrm{c} 1$. In both the subfigures, the molecule in black box is the scaffold used for conditional generation. (a, b) 8 random generated molecules having the same scaffold as scaffold 1 and 2 respectively. 

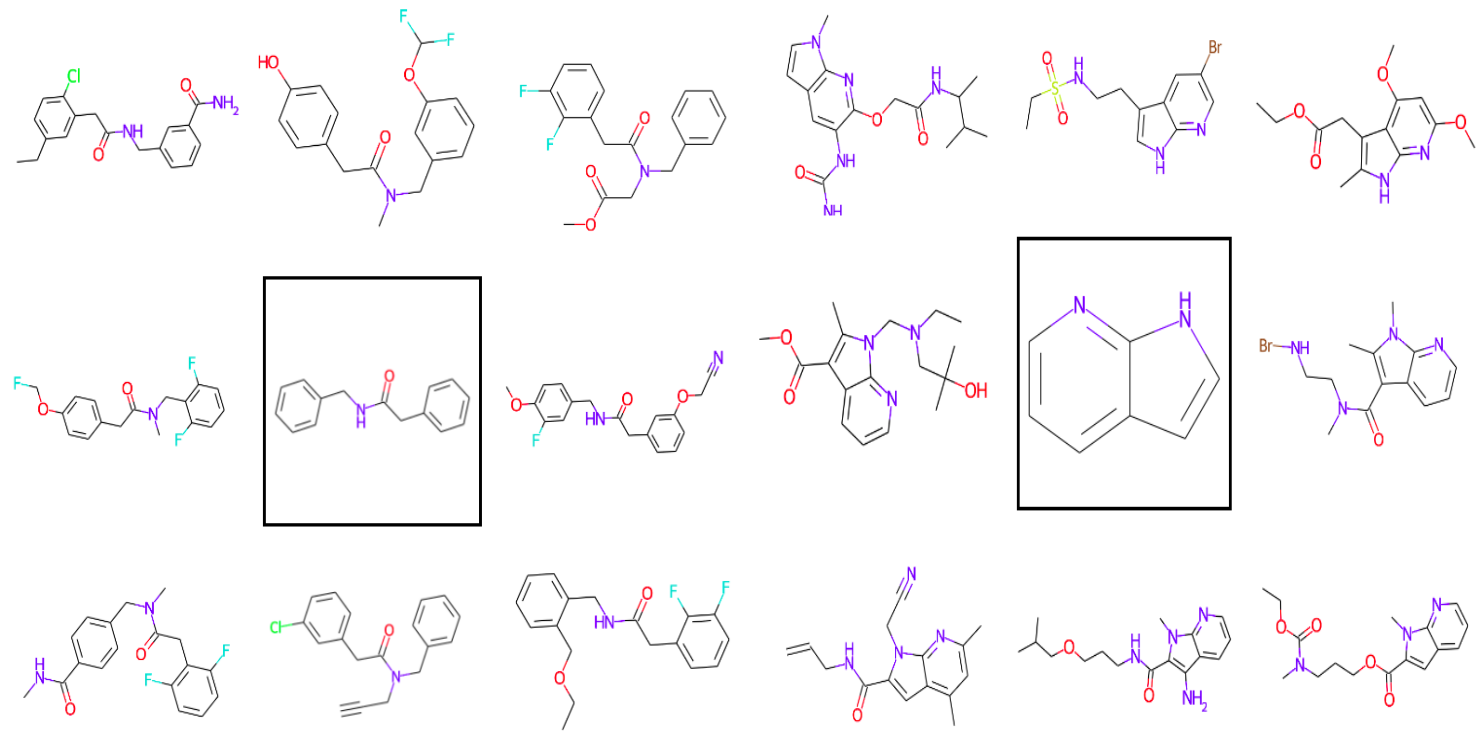

(a) Scaffold 1, $\log \mathrm{P}$

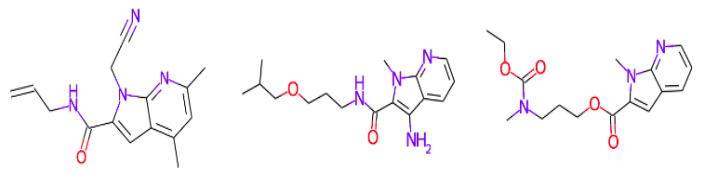

(b) Scaffold 2, $\log \mathrm{P}$
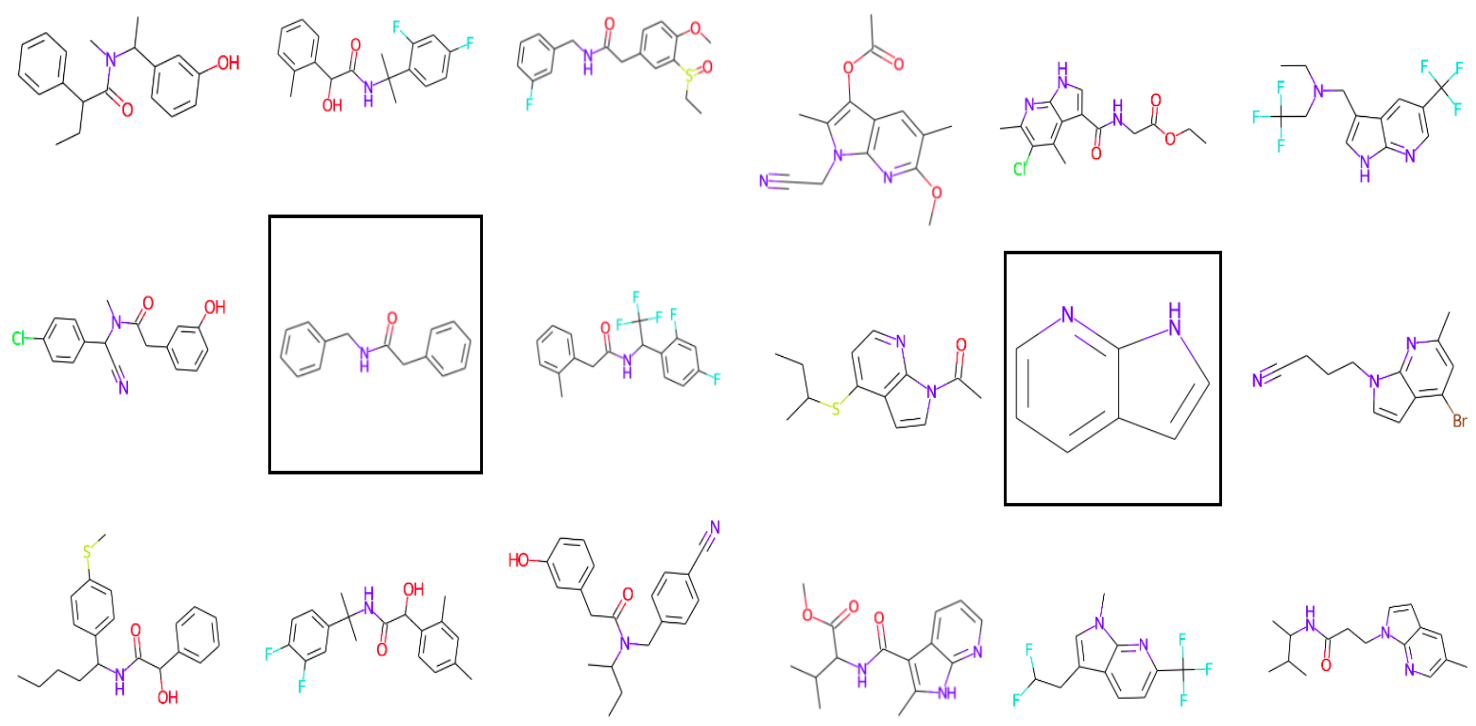

(c) Scaffold 1, SAS

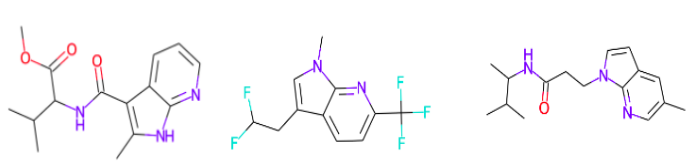

(d) Scaffold 2, SAS

Figure S3: Scaffold 1: $\mathrm{O}=\mathrm{C}(\mathrm{Cc} 1 \mathrm{ccccc} 1) \mathrm{NCc1} 1 \mathrm{cccc} 1$. Scaffold 2: $\mathrm{c} 1 \mathrm{cnc} 2[\mathrm{nH}] \mathrm{ccc} 2 \mathrm{c} 1$. In all the subfigures, the molecule in black box is the scaffold used for conditional generation. (a, b) Conditioned on scaffold as well as $\log \mathrm{P}=2$. (c, d) Conditioned on scaffold as well as $\mathrm{SAS}=2.75$. 